\title{
Heterometallic Cr-Mn Complexes Containing Cyanide and Oxalate Bridges
}

\section{Yuan-Zhu Zhang, Zhe-Ming Wang, Song Ga**}

* Beijing National Laboratory for Molecular Sciences, State Key Laboratory of Rare Earth

Materials Chemistry and Applications, College of Chemistry and Molecular Engineering, Peking University, Beijing 100871, P. R. China

Fax: (+86)-10-62751708

E-mail: gaosong@pku.edu.cn

\section{Support Information}

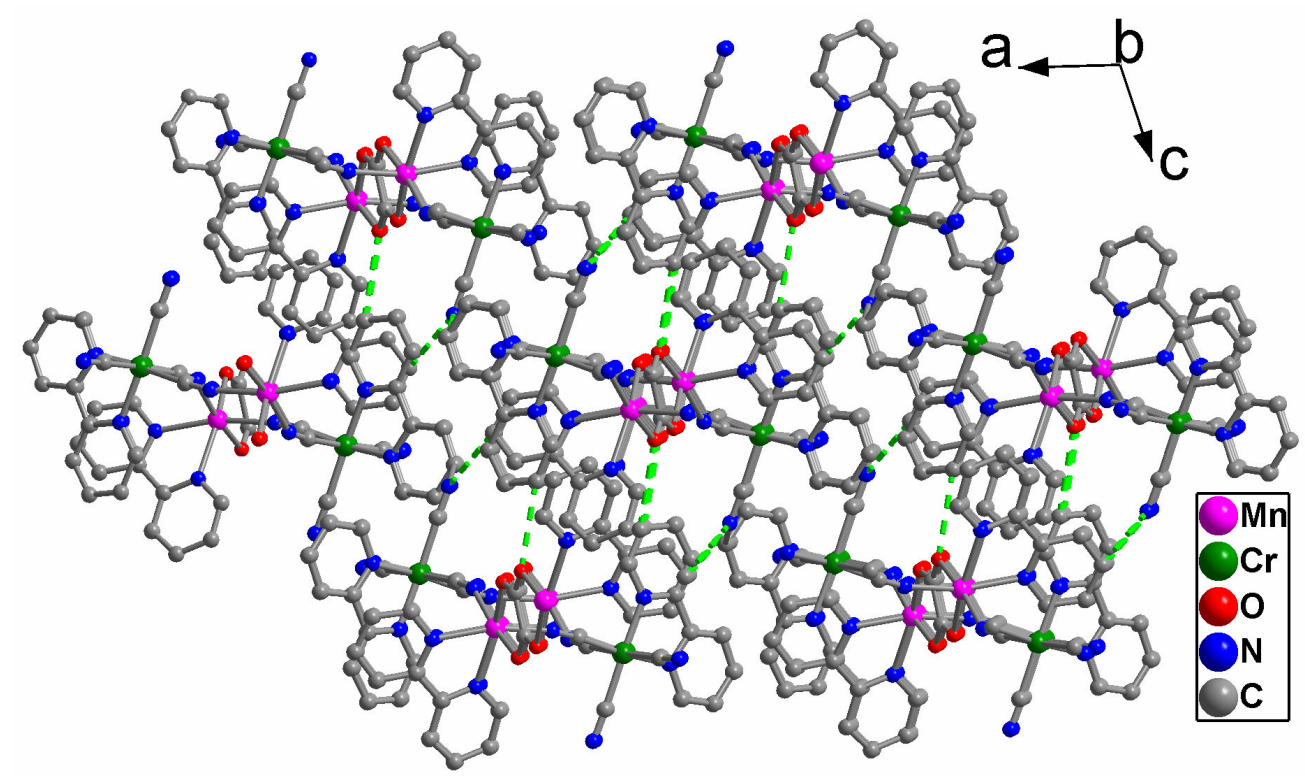

Figure S1 Packing of the cluster-based chains for 3 through van der Waals contacts and weak Hbonds. 


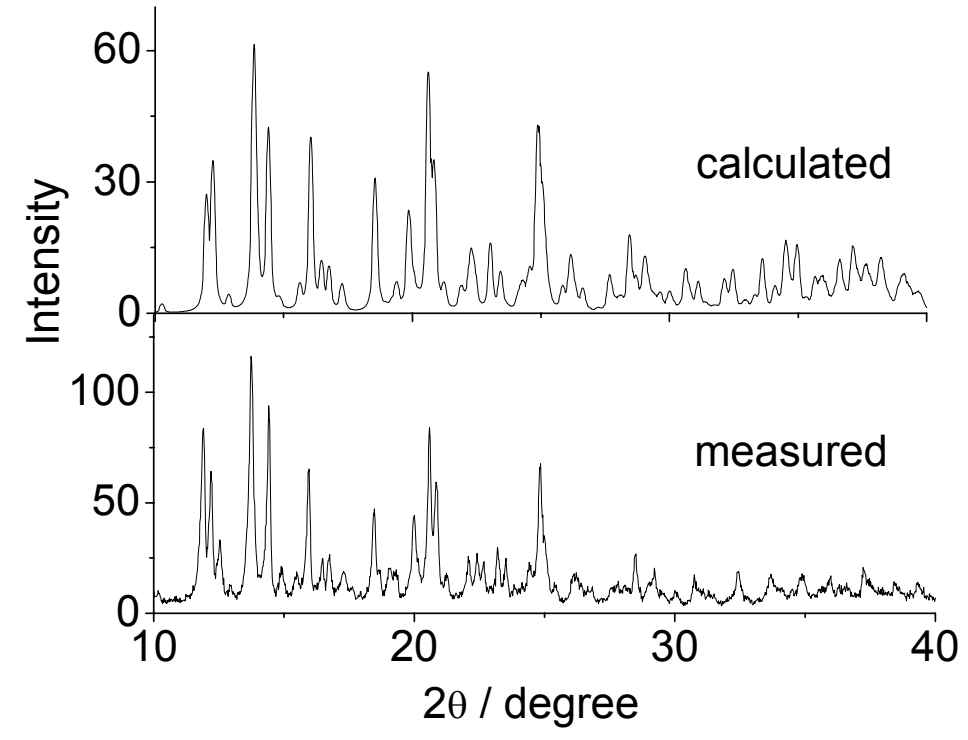

Figure S2 The calculated (upper) and measured (down) XRD patterns for 3. XRD data were collected at room temperature with a Rigaku Dmax-2000 diffractometer equipped with a $\mathrm{Cu} \mathrm{K} \alpha$ radiation source $(\lambda=0.15418 \mathrm{~nm})$. 